\title{
MSFC INVESTIGATIONS OF BETA CLOTH DARKENING DUE TO ULTRAVIOLET RADIATION INTERACTIONS
}

\author{
Rachel R. Kamenetzky \\ ED31 \\ Marshall Space Flight Center, AL 35812 \\ (256) 544-1089 \\ rachel.kamenetzky@msfc.nasa.gov
}

\author{
Miria M. Finckenor \\ ED31 \\ Marshall Space Flight Center, AL 35812 \\ (256) 544-9244 \\ miria.finckenor@msfc.nasa.gov \\ Senior Member, AIAA
}

\begin{abstract}
A common component of multi-layer insulation blankets is beta cloth, a woven fiberglass cloth impregnated with Teflon. It is planned for extensive use on the International Space Station (ISS). The Environmental Effects Group of the Marshall Space Flight Center Materials, Processing and Manufacturing Department has investigated the impact of atomic oxygen (AO) and ultraviolet (UV) radiation on the optical properties of plain and aluminized beta cloth, both in the laboratory and as part of long-duration flight experiments. These investigations indicate that beta cloth was susceptible to darkening in the presence of UV radiation, dependent on the additives used. The presence of AO countered some, if not all, of the UV degradation.
\end{abstract}

\section{INTRODUCTION}

The Environmental Effects Group has several facilities for the study of space environmental effects on materials. The Atomic Oxygen Beam Facility (AOBF) and the Atomic Oxygen Drift Tube System (AODTS) have been used to determine the effect of AO on a number of materials. Solar simulation facilities are also available for UV radiation exposure of materials. Full details of the capabilities of the Environmental Effects Group (formerly known as the Physical Science and Environmental Effects Branch) may be found in reference 1. This report discusses individual exposures to $\mathrm{AO}$ and $\mathrm{UV}$, as well as synergistic exposure in the laboratory.

Actual exposure to the space environment is preferable but not always possible when studying candidate spacecraft materials. Comparison of flight results to ground simulations gives confidence to the simulation method and the durability of the material for longer exposures. Beta cloth has been flown on several materials flight experiments. Results from the Long Duration Exposure Facility (LDEF) as well as the more recent International Space Station Risk Mitigation Experiments gives confidence in beta cloth's durability in the low Earth orbit environment.

\section{LABORATORY INVESTIGATIONS}

Qualifying beta cloth for use on the International Space Station required testing in AO and UV environments and ensuring the stability of the optical properties and mechanical integrity. Optical properties were measured using an AZ Technology Laboratory Portable Spectroreflectometer (LPSR) to measure solar absorptance and either a Gier-Dunkle DB100 or an AZ Technology TEMP 2000 to measure infrared emittance. 
Laboratory investigations of beta cloth at MSFC (ref. 2) showed that beta cloth may noticeably darken when exposed to UV radiation alone, and the amount of darkening varies by UV source and beta cloth batch. Degradation is also dependent on lack of oxygen, as beta cloth samples did not darken when exposed to UV radiation in air but did darken when the same test was repeated in vacuum.

Plain (unaluminized) and aluminized beta cloth were exposed to 8,000 ESH of vacuum UV radiation (130 nm wavelength peak) and had a yellowed appearance post-test. Optical property data from this test may be found in Table 1 .

Table 1. Vacuum UV Effects on Optical Properties

\begin{tabular}{|c|c|c|c|c|}
\hline \multirow{2}{*}{ Material } & \multicolumn{2}{|c|}{ Solar Absorptance } & \multicolumn{2}{c|}{ Infrared Emittance } \\
\cline { 2 - 5 } & Control & $\begin{array}{c}\text { Post-Test } \\
8000 \text { ESH }\end{array}$ & Control & $\begin{array}{c}\text { Post-Test } \\
8000 \text { ESH }\end{array}$ \\
\hline Unaluminized & 0.19 & 0.22 & 0.90 & 0.90 \\
\hline Aluminized & 0.31 & 0.33 & 0.91 & 0.90 \\
\hline
\end{tabular}

Without the synergistic presence of $\mathrm{AO}$, beta cloth appears to darken. An aluminized beta cloth sample was exposed to approximately $700 \mathrm{ESH}$ of enhanced UV radiation from 250 to $400 \mathrm{~nm}$ in a solar simulator, darkening it to a solar absorptance value of 0.37 . It was then bleached by $2.1 \times 10^{20}$ atoms $/ \mathrm{cm}^{2}$ of thermal energy AO to a solar absorptance value of 0.31 .

Near-UV sources appeared to have more effect on beta cloth than vacuum UV sources. UV darkening also appeared to vary batch by batch. As few as $300 \mathrm{ESH}$ of enhanced UV radiation exposure visibly darkened or yellowed beta cloth. The key factor in darkening appeared to be the use of a polysiloxane, which is added for flexibility. This concurs with research performed at other NASA centers (ref. 3). Aluminized beta cloth with $2 \%, 0.22 \%$, and no silicone were exposed to $800 \mathrm{ESH}$ of xenon lamp UV radiation with a cut-off at $180 \mathrm{~nm}$. Solar absorptance values for this research are given in Table 2.

Table 2. UV Effects on Solar Absorptance of Aluminized Beta Cloth

\begin{tabular}{|c|c|c|c|}
\hline ESH & $2.0 \%$ Silicones & $0.22 \%$ Silicones & Silicone-Free \\
\hline 0 & 0.29 & 0.31 & 0.32 \\
\hline 200 & 0.30 & 0.32 & 0.32 \\
\hline 400 & 0.32 & 0.32 & 0.32 \\
\hline 800 & 0.34 & 0.32 & 0.33 \\
\hline
\end{tabular}

LaRC UV Test, NASA TM-104748, Koontz et al.

Based on available data at that time, we recommended ordering beta cloth without the polysiloxane and performing lot testing for ISS beta cloth prior to MLI blanket assembly with a minimum of 500 ESH of UV radiation.

Koontz, Jacobs, and Le also note that the beta cloth was sandblasted on one side prior to aluminization. The manufacturer has changed the preparation technique prior to aluminization since these tests were performed. The current preparation process uses a film that is heat-bonded to the beta cloth, making it easier to apply the aluminization. 


\section{FLIGHT RESULTS}

The first flight experiment included in this study was the Long Duration Exposure Facility. One experiment, the Transverse Flat-Plate Heat Pipe Experiment (ref. 4) used plain beta cloth as part of its MLI blankets. This experiment was $22^{\circ}$ off the ram direction, receiving $8.43 \mathrm{x}$ $10^{21}$ atoms $/ \mathrm{cm}^{2}$ of $\mathrm{AO}$ and 8,680 equivalent sun-hours (ESH) of solar $\mathrm{UV}$ radiation. Though the beta cloth lost Teflon due to AO erosion, the fiberglass weave was tight enough to prevent any AO damage to underlying layers. No apparent darkening occurred, and optical properties remained stable.

Aluminized beta cloth was flown on three long-duration flight experiments, the Passive Optical Sample Assemblies - I and II and the Optical Properties Monitor (OPM) . POSA-I, POSA-II and OPM are Phase I Risk Mitigation Experiments for ISS and were attached to the Mir/Shuttle Docking Module (fig. XX) of the Mir Space Station by EVA. Mir is in a $390 \mathrm{~km}$ orbit at $51.6^{\circ}$ inclination. POSA-I consisted of a specially designed "suitcase" carrier with two identical sets of samples, oriented so that one set faced the Mir core, and the other set faced space. POSA-II was identical to POSA-I in the suitcase design but carried a completely different set of samples and was oriented $45^{\circ}$ off the ram direction.

POSA-I and POSA-II were exposed to the Mir-induced and natural space environment for eighteen months. Both experiments flew 6" x 6" MLI blankets identical to the ISS configuration. MLI blankets on POSA-I used two different threads, one of Nomex, the other of beta glass and Teflon. MLI blankets on POSA-II used a beta glass and nylon thread which had a CVCM of $0.12 \%$, a borderline failure of the strict molecular contamination requirements for ISS cleanliness. Yellowing of the beta cloth was noted around this thread.

The POSA-I MLI blanket facing the Mir core received approximately $7 \times 10^{19}$ atoms $/ \mathrm{cm}^{2}$ of $\mathrm{AO}$ and $413 \mathrm{ESH}$ of solar UV. The beta cloth on this blanket had a $3.4 \%$ increase in solar absorptance and no significant change in infrared emittance. The POSA-I MLI blanket facing space were contaminated on-orbit with silicone photodeposition (ref. 5) and saw a $8.9 \%$ increase in solar absorptance. The percent change data for these samples has been corrected for instrumentation drift with pre-flight control sample measurements.

The POSA-II MLI blanket in the nominal ram direction received $2.1 \times 10^{20}$ atoms $/ \mathrm{cm}^{2}$ of AO (ref. 6) and 576 ESH of solar UV. This blanket was also contaminated with some silicone though not of the same magnitude as POSA-I. Visible splash areas on POSA-II surfaces facing the Space Shuttle indicate contamination by a water/waste dump. Despite the level of man-made contamination, solar absorptance increased only 3.3 to $6.1 \%$ for this blanket, dependent on proximity to the beta glass and nylon thread and the amount of contamination. The POSA-II MLI blanket in the nominal wake direction also increased in solar absorptance by 6.1 to $8.6 \%$. The exposure for the wake samples was $8.2 \times 10^{19}$ atoms $/ \mathrm{cm}^{2}$ of AO and approximately $500 \mathrm{ESH}$ of solar UV. No significant change in infrared emittance was noted for any of the samples. 